\title{
Solar disinfection: use of sunlight to decontaminate drinking water in developing countries
}

A safe or reliable year-round supply of drinking water remains a problem for at least one-third of the population of developing countries [1], as effective filtration or chlorination are often beyond the financial means of the community. Boiling water before drinking is not always feasible, especially if fuel is expensive (financially or environmentally) or labour intensive to collect. Burning carbon-based fuels indoors in poorly ventilated dwellings can also have a significant impact on lung disease. A water treatment process that requires virtually no initial expense and absolutely no running cost would be of inestimable value to those most at risk of water-borne disease. This is the essential appeal of solar disinfection: to use a combination of irradiation by direct sunlight and solar heating to kill the waterborne pathogens in contaminated drinking water.

In one form of solar disinfection, contaminated water is pumped through a long series of transparent pipes that are arranged in a panel facing the sun. While this can supply the needs of an entire village, the main disadvantages are the cost and availability of the raw materials needed to construct the pump systems and solar panel. A cheaper and simpler alternative is to store drinking water in transparent containers, which are then placed in direct sunlight. The attraction of this method is that all that is needed is a sealable, transparent - glass or plastic - bottle or bag. In trials in Kenya, uncoloured, transparent, plastic bottles (cola, soft drinks, mineral water, etc.) were favoured because they were easy to obtain [2]. Most of the containers were donated by urban primary school pupils who had retrieved them from their own domestic refuse.

The inactivation mechanism depends on the ambient solar conditions, but involves optical and thermal processes. The effect of sunlight on bacterial pathogens is well documented. UV-A and early visible wavelength regions $(320-450 \mathrm{~nm})$ of the sunlight spectrum are absorbed by photosensitisers that become electronically excited and react with neighbouring oxygen molecules. This leads to the production of highly reactive oxygen species that cause strand breakage and base changes in DNA [3]. Strand breakage is usually lethal, while base changes may result in a block in replication and other mutagenic effects [4]. Sublethal optical doses may render the bacteria temporarily inactive or non- culturable, and such damage may be corrected by DNA repair mechanisms that are active in most living cells. Therefore the optical inactivation process is sometimes reversible, particularly for bacteria in the stationary phase of growth. Little is known about the pathogenicity of viable but non-cultivable organisms.

During solar exposure, water temperatures within the bottles can reach $55^{\circ} \mathrm{C}$. Even higher temperatures can be achieved if the base or back of the bottle is blackened to enhance heat transfer through infrared absorption. The pasteurising effect of such temperatures over a period of hours causes complete and irreversible inactivation of most bacterial pathogens $[5,6]$, except thermotolerant or spore-forming species [7]. No thermal inactivation has been reported for water temperatures $<40^{\circ} \mathrm{C}$. Highly turbid or unfiltered water may not support any significant optical inactivation, because the sunlight is completely absorbed within the first few millimetres of water. However, turbidity usually increases the maximum water temperature achieved within the bottle.

Strong synergy is observed when the optical and thermal inactivation processes are combined during solar disinfection. Optical inactivation rates for Escherichia coli, rotavirus and bacteriophage $\mathrm{f} 2$ increase by factors of up to 3.6 when water temperature is raised from $20^{\circ} \mathrm{C}$ to $40^{\circ} \mathrm{C}$ [1]. The optical and thermal environments established within water samples during batch-process solar disinfection exposures inactivate $E$. coli, Salmonella enterica serotypes Typhi, Paratyphi and Enteritidis, Shigella flexneri, Pseudomonas aeruginosa [3], Enterococcus faecalis [8] and Vibrio cholerae [9].

Viruses studied so far (bovine rotavirus, encephalomyocarditis virus, bacteriophage $\mathrm{f} 2$ and polio virus $[1,9])$ are particularly sensitive to the optical inactivation mechanisms that result from solar exposure. One reason put forward is that, unlike many bacteria, they are unable to repair optically induced damage to their DNA.

Nothing has been reported yet on the effects of solar disinfection on other water-borne viral pathogens such as Norwalk virus, hepatitis A or hepatitis E, or on 
protozoa and helminths. If the water temperature reaches $56^{\circ} \mathrm{C}$, cysts of Giardia lamblia, G. muris and Entamoeba histolytica are thermally inactivated after $10 \mathrm{~min}$ [10]. Unfortunately, these temperatures can be achieved in 1-L samples only if there is prolonged strong sunshine $(>4 \mathrm{~h})$ or the basic solar disinfection technique is enhanced to boost the thermal effect, e.g., by the use of half-blackened bottles, a solar box or parabolic cookers.

Well-documented field trials of solar disinfection are few and far between. Several controlled, randomised field trials have been conducted in the Maasai communities in Kajiado province, Kenya over the past 3 years [2]. All children in alternate households were randomised to drink water from transparent plastic bottles, either kept on the roof or indoors (control). The randomisation by alternate household ensured that the protocol was realistic, simple to implement and automatically compensated for variations in water quality within the trial area.

In the first trial, 206 children aged 5-16 years were randomised and followed fortnightly for 12 weeks. Children consuming solar-disinfected water had a $10 \%$ reduction in the incidence of diarrhoea, including a $24 \%$ reduction in the incidence of severe diarrhoea over the study period. A subsequent 1-year study of 349 children aged $<5$ years found a similar reduction in risk. The results confirmed what the mothers themselves had observed: that there was a modest reduction in the risk of diarrhoea when children drank solar disinfected water. This has led to the adoption of solar disinfection by the communities in which the trial was run. It remains to be seen if the custom will persist, but at least the transition from scientific knowledge to changed health behaviour has been made to some extent. In addition, the occurrence of a cholera outbreak in the area has provided a valuable opportunity to examine the protective effect of solar-disinfected drinking water on the risk of cholera in young children. The results of this study are currently being analysed.

Solar disinfection offers a worthwhile reduction in disease risk under 'real life' conditions, but is not a panacea. A central problem is that people cannot usually be relied on to follow laboratory protocols. In practice they may not leave the water in the sun for long enough before drinking it, and they will probably also drink water directly from water holes or other sources. In addition, contaminated water is only one of the causes of diarrhoeal disease, which is often the main public health problem in areas where solar disinfection offers potential benefits.

Solar disinfection offers no protection against chemical contaminants in drinking water and concerns have been raised about health risks associated with chemicals leached from the plastic containers by prolonged photo- degradation. Most plastic drink containers are presently made from polyethylene terephthalate. The compounds that could potentially leach from such bottles are acetaldehyde, terephthalic acid, dimethylterephthalate and ethylene glycol. Terephthalic acid and dimethylterephthalate are genotoxic, but they are insoluble in water, and the chances of them leaching into the water, even during solar disinfection, are minimal. Ethylene glycol is more likely to leach into the water, as it is more water-soluble (Dr M. T. Kelly, Université, Montpellier, personal communication). Preliminary comparisons of shaded controls and similar bottles exposed to Malaysian tropical sun for 3 consecutive months have so far failed to detect any of these compounds.

Although solar disinfection can be a labour-intensive process and may not be universally appropriate throughout all developing countries, there are circumstances in which it may be the only alternative to untreated drinking water. Reed [8] identified these as:

1. The provision of treated water for people in rural villages and in urban shanty communities, who may have access only to sewage-contaminated surface water.

2. The provision of decontaminated water to widely dispersed rural populations, mountainous locations or semi-nomadic communities, where a piped water supply may be impractical or where chemical treatment is too costly.

3. Emergency water supplies for refugees and in war zones, where conventional water supplies may be unavailable, disrupted or inoperative.

4. Short-term treatment in response to a specific contamination event such as storm-water or flooding.

5. Short-term treatment of a source contaminated with pathogenic bacteria; e.g., during an outbreak of cholera or bacterial diarrhoea, in the absence of an alternative treatment or a suitable water source.

6. The provision of treated drinking water for babies or infants, as they are most at risk of death due to diarrhoeal disease.

7. The preparation of decontaminated water for oral rehydration solution where no reliable safe water supply exists.

Other advantages can result from solar disinfection. In endemic areas, schistosomiasis (bilharzia) can be contracted from drinking water containing the cercarial stage of schistosome worms, although this is not the most common route of infection. Cercariae lose the ability to penetrate skin or mucosa within $48 \mathrm{~h}$ of being shed by their aquatic snail host. Solar disinfection can therefore remove one transmission route of schistosomiasis if drinking water is allowed to stand for 2 full days before being consumed. Another advantage of storing drinking water in transparent, rather than opaque, containers is that the risk of ingesting leeches is greatly reduced, as it is immediately apparent if they 
are present in the water (Dr T. O'Dempsey, personal communication).

No study has found that solar exposure makes water quality worse, even under overcast conditions in temperate climates. Ultimately, communities with no established water treatment facility have nothing to fear or lose from using solar disinfection. The basic apparatus can be obtained from most household refuse. The fuel costs nothing!

K. G. McGUIGAN, T. M. JOYCE* and R. M. CONROY $\dagger$ Departments of Physics, *Tropical Medicine and $\uparrow$ Epidemiology, Royal College of Surgeons in Ireland 123 St Stephens Green, Dublin 2, Ireland Corresponding author: Dr K. G. McGuigan (e-mail: kmcguigan@rcsi.ie)

\section{References}

1. Wegelin M, Canonica S, Mechsner K, Fleischmann T, Pesaro F, Metzler A. Solar water disinfection: scope of the process and analysis of radiation experiments. Aqua 1994; 43: 154-169.

2. Conroy RM, Elmore-Meegan M, Joyce T, McGuigan KG, Barnes J. Solar disinfection of drinking water and diarrhoea in Maasai children: a controlled field trial. Lancet 1996; 348 : 1695-1697.

3. Acra A, Jurdi $M$, Mu'allem $H$, Karahagopian $Y$, Raffoul $Z$. Water disinfection by solar radiation. International Development Research Centre, Ottawa, Canada. 1990 IDRC-TS66e.

4. Barer MR, Gribbon LT, Harwood CR, Nwoguh CE. The viable but non-culturable hypothesis and medical microbiology. Rev Microbiol 1993; 4: 183-191.

5. Joyce TM, McGuigan KG, Elmore-Meegan M, Conroy RM. Inactivation of fecal bacteria in drinking water by solar heating. Appl Environ Microbiol 1996; 62: 399-402.

6. McGuigan KG, Joyce TM, Conroy RM, Gillespie JB, ElmoreMeegan M. Solar disinfection of drinking water contained in transparent plastic bottles: characterizing the bacterial inactivation process. J Appl Microbiol 1998; 84: 1138-1148.

7. Fjendbo-Jorgensen A, Nohr $K$, Sorensen $H$, Boisen $F$ Decontamination of drinking water by direct heating in solar panels. J Appl Microbiol 1998; 85: 441-447.

8. Reed RH. Solar inactivation of faecal bacteria in water: the critical role of oxygen. Lett Appl Microbiol 1997; 24: 276-280.

9. Sommer B, Marino A, Solars YL et al. SODIS - an emerging water treatment process. $J$ Water SRT-Aqua 1997; 46: 127-137.

10. Ciochetti DA, Metcalf RH. Pasteurization of naturally contaminated water with solar energy. Appl Environ Microbiol 1984; 47: 223-228. 